\title{
Immunoproteomics approach for prediction of antigenic epitope of Tertiapin from Apis mellifera
}

Gomase V. S., Phadnis A. C. and Ghatak A.A.

Department of Bioinformatics, Padmashree Dr. D. Y. Patil University, Plot No-50, Sector-15, CBD Belapur, Navi Mumbai, 400614, India, Mail- virusgene1@yahoo.co.in, Mobile- +91-9987770696, www.bioinfo.in

\begin{abstract}
The bee venom is used for treating a wide variety of disease conditions. It has also been found to be a strong immunological agent, stimulating the body's protective mechanisms against disease. The major treatment is gene therapy or recombinant DNA vaccines involved targeting multiple antigenic components to direct and empower the immune system to protect the host from infection. Limitation of therapy to the treatment of patients suffering from various adverse reaction and contraindications are always experienced. Tertiapin is a neurotoxin from the honeybee venom. It interacts specifically with calmodulin in the presence of Ca2+. Antigenic epitopes on Tertiapin protein of Apis mellifera (honey bee) is important determinants sites for protection against disorders. As our knowledge of the immune responses to a protein antigen progressed, it became clear that the whole protein is not necessary for raising the immune response, but small segments (NCNRIIIPHMCWK, 4-16) of tertiapin protein called the antigenic determinants or the epitopes are sufficient for eliciting the desired immune response. Immunization cassettes should be capable of immunizing of broad immunity against both humoral and cellular epitope thus giving vaccines the maximum ability to deal with Tertiapin protein of Apis mellifera immune escape. We have predicted a successful immunization strategy.
\end{abstract}

Keywords- Tertiapin, apitherapy, Antigenic epitope, Hydrophobicity, Hydrophilicity

\section{Introduction}

Apitherapy, or bee therapy, is the use of products of the common honeybee for therapeutic purposes. Honeybee venom, bee pollen, raw honey, royal jelly, and propolis are the products generally considered to have medicinal effects. These products are said to be effective against a wide range of ailments, from arthritis and chronic pain to multiple sclerosis and cancer, although few scientific studies have as yet proved their benefits. Bee venom is a potent anti-inflammatory antioxidant, antifungal, antibacterial, and possesses radioprotectant actions. It has been found to exert powerful actions as an antibacterial agent, anti-inflammatory, antiarthritic antirheumatic, neurodegenerative disease, as a cardiotonic, an antioxidant, and as a diaphoretic and diuretic. It has also been found to be a strong immunological agent, stimulating the body's protective mechanisms against disease. The Eclectic Botanical physicians considered it to be a potent alterative.

\section{Tertiapin}

Tertiapin is a neurotoxin from the honeybee venom. It interacts specifically with calmodulin in the presence of $\mathrm{Ca} 2+$. Miroshnikov, et al. 1983 was studied using calmodulin-cAMP phosphodiesterase system. Tertiapin does not affect the unstimulated basal activity of phosphodiesterase. However, it totally inhibits the enzyme-activating capacity of calmodulin. Analysis of the dose-dependent activation of phosphodiesterase by calmodulin in the presence of tertiapin indicated that inhibition is caused by the interaction of two tertiapin molecules with calmodulin ( $\mathrm{Kd} 2$ microM). The data obtained suggest that the toxic effect of tertiapin in nervous tissue is mediated by blockade of calmodulin function [1].
The cells contain phosphodiesterase of two types. One of them possesses a high affinity for cyclic AMP. The cell regulation is phosphodiesterase with low affinity for the substrate and its activity is modulated by the calmodulin-Ca2+ complex, cyclic GMP and $\mathrm{NAD}+$ at micromolar concentrations. The effect on the phosphodiesterase system of a "quick" messenger, $\mathrm{Ca} 2+$ and "slow" messengers, cyclic GMP and NAD+, has the same consequences. The functional significance of such an activity modulation may be the necessity to maintain the conditions for phosphodiesterase functioning when $\mathrm{Km}$ much greater than cyclic AMP and the reaction rate are directly proportional to the substrate concentration. Studies of PC-12 cells revealed a chronic effect of phosphodiesterase activity change under the action of staphylococcal enterotoxin A. This protein induces differentiation of PC-12 cells and possesses a NAD+-glycohydrolase activity. As a result, the enzyme activity falls, cyclic AMP level increases and cell differentiation starts. The activity of soluble phosphodiesterase of PC-12 cells also decreases under the effect of two neurotoxins from bee venom, melittin and tertiapin. Both the toxins at concentration of 10 microM completely block calcium regulation of the enzyme. Inhibition of $\mathrm{Ca} 2+$ action is achieved as the result of the activated calmodulin molecule [2]. Tertiapin is a small protein derived from honeybee venom. It inhibits the GIRK1/4 and ROMK1 channels with nanomolar affinities. Methionine residue 13 in TPN interacts with residue F148 in the channel, located just outside of the narrow region of the ROMK1 pore [3]. A recent study indicated that tertiapin is a potent blocker of certain types of inwardly rectifying $\mathrm{K} \quad(+)$ (Kir) channels. Tertiapin fully inhibited acetylcholine (1 microM)-induced muscarinic $\mathrm{K}(+)(\mathrm{K}(\mathrm{ACh}))$ channel currents in atrial myocytes. At the single-channel level, tertiapin inhibited the 
$\mathrm{K}(\mathrm{ACh})$ channel from the outside of the membrane by reducing the $\mathrm{NP}(0)$ ( $N$ is the number of functional channels, and the $P(0)$ is the open probability of each channel) without affecting the single-channel conductance or fast kinetics. Therefore, tertiapin potently and selectively blocks the $\mathrm{K}(\mathrm{ACh})$ channel in cardiac myocytes in a receptor- and voltageindependent manner [4]. Tertiapin is a novel pharmacological tool to identify the functional role of the $\mathrm{K}(\mathrm{ACh})$ channel in the parasympathetic regulation of the heart beat. Tertiapin, blocks a G-protein-gated channel (GIRK1/4) and the ROMK1 channel with nanomolar affinities, but a closely related channel, IRK1, is insensitive to tertiapin. Mutagenesis studies show that tertiapin inhibits the channel by binding to the external end of the ion conduction pore [5]. In this experiment we found the antigenic epitope for tertiapin from Apis mellifera [6]

Materials and Methods 1. Database searching.

Genomic databases are used to store the vast amount of information issuing from the genome projects. We analyze the Tertiapin protein of Apis mellifera [7-9].

\section{Prediction of antigenic peptides}

Prediction of antigenic peptide program predicts those segments from within a Tertiapin protein of Apis mellifera (honey bee) sequence that are likely to be antigenic by eliciting an antibody response. Antigenic peptides are determined using the method of Kolaskar and Tongaonkar (1990) [10]. Predictions are based on a table that reflects the occurrence of amino acid residues in experimentally known segmental epitopes. Segments are only reported if the have a minimum size of 8 residues. The reported accuracy of method is about $75 \%$.

Method specification -

Program - Prediction antigenic peptides.

Method - Antigen prediction.

Protein sequence - Tertiapin protein of Apis

mellifera (honey bee)

Format - Raw sequence

\section{Finding the location in solvent accessible regions}

For setting the solvent accessible regions in protein, type of plot determine the hydrophobic scale and it is utilized for prediction. Input Tertiapin protein of Apis mellifera sequence and obtain plots that characterize its hydrophobic [11] and hydrophilic [12] character, which may be useful in predicting membranespanning domains, potential antigenic sites and regions that are likely exposed on the protein surface.

Method specification -

Program- Protein Hydrophobicity Plots

Method- Kyte-Doolittle, Hopp-Woods

Protein sequence - Tertiapin protein of Apis mellifera (honey bee)

\section{Format- Raw sequence}

\section{Results}

Sequence of Tertiapin protein of Apis mellifera (honey bee) is as follows:

ALCNCNRIIIPHMCWKKCGKK
Tertiapin protein of Apis mellifera (honey bee)
sequence is 21 amino acids residues long.

\section{Prediction of Antigenic peptides}

Antibodies find multiple applications in a variety of areas including biotechnology, pharmaceuticals for diagnosis and indeed they are one of the most powerful tools for life science research. These are directed against protein antigens and can recognize either linear or native three-dimensional (3D) epitopes. Antibodies that recognize 3D epitopes require the use of whole native protein as immunogens. Due to various technical reasons this is not always a choice. In such cases, immunization with peptides is the alternative. Antibodies generated in this manner will recognize linear epitopes, and they might or might not recognize the source native protein, but yet they will be useful for standard laboratory applications such as western blots.

Antigenic plot for Tertiapin protein of Apis mellifera (honey bee) sequence

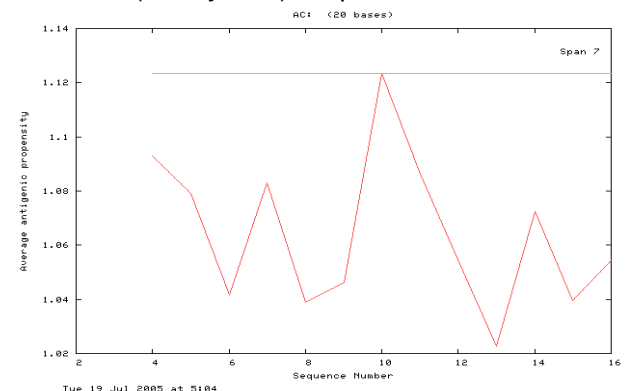

Figure 1 - Antigenic determinant plot. X-axis contains sequence number and $y$-axis contain average antigenic propensity. There is one (1) antigenic determinant in sequence. The highest pick at start position is 4 to 16 . The sequence is- NCNRIIIPHMCWK. The average propensities for the whole protein is above 1.0697, however if propensities more than 1.0 are potentially antigenic.

Table 1- There is one (1) antigenic determinants site in Tertiapin protein sequence

\begin{tabular}{|c|c|c|c|}
\hline $\begin{array}{c}\text { Sr. } \\
\text { No. }\end{array}$ & $\begin{array}{c}\text { Start } \\
\text { Position }\end{array}$ & Sequence & $\begin{array}{c}\text { End } \\
\text { Position }\end{array}$ \\
\hline 1 & 4 & NCNRIIIPHMCWK & 16 \\
\hline
\end{tabular}

\section{Solvent accessible regions}

Kyte-Doolittle is a widely applied scale for delineating hydrophobic character of a protein. Regions with values above zero (0) are hydrophobic in character. Hopp-Woods scale was designed for predicting potentially antigenic regions of polypeptides. Values greater than zero (0) are hydrophilic and thus likely to be exposed on the surface of a folded 
protein. Window size refers to the number of amino acids examined at a time to determine a point of hydrophobic character. Window size can be varied from 5 to 25 (default 7) and one should choose a window that corresponds to the expected size of the structural motif under investigation. Window size of 5-7 is good for finding hydrophilic regions that are likely exposed on the surface and may possibly be antigenic. Window size of 19-21 will make hydrophobic, membrane-spanning domains stand out rather clearly (typically $>1.6$ on the Kyte-Doolittle scale). The demonstration protein is the glycoprotein of vesicular stomatitis virus, which has a transmembrane domain near the carboxy-terminus (right side of plot).

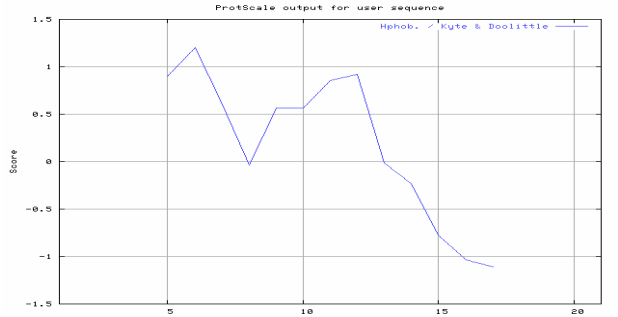

Figure 2- Hydrophobicity plot of Tertiapin protein of Apis mellifera. The sequence isNCNRIIIPHMCWK in the plot in above the zero indicates it is hydrophobic in nature. Input a protein sequence and obtain plots that characterize its hydrophobic character, which may be useful in predicting membranespanning domains, potential antigenic sites and regions that are likely exposed on the protein's surface.

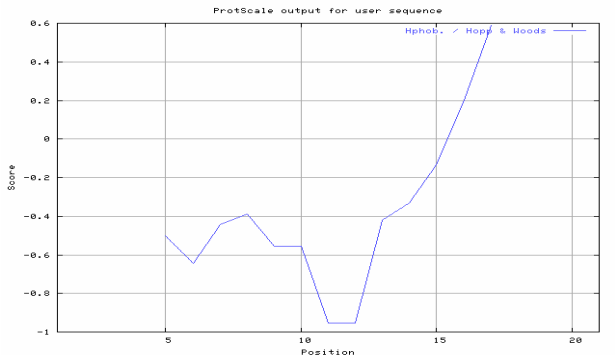

Figure 3 - Hydrophilicity plot Tertiapin protein of Apis mellifera. The sequence isNCNRIIIPHMCWK in the plot in below the zero indicates it is hydrophobic in nature.

\section{Structural Analysis}

Structural analysis of tertiapin protein shows the helical region in (12-19 $\mathrm{AA}$ ) and turn region in (5-9 AA). Predicted antigenic epitope is form partially helical region. Methionine amino acid is present at 13 positions in helical region. Helical region is form from 'HMCWKKCG' this amino acids. Turn is form from 'CNRII' this amino acids.

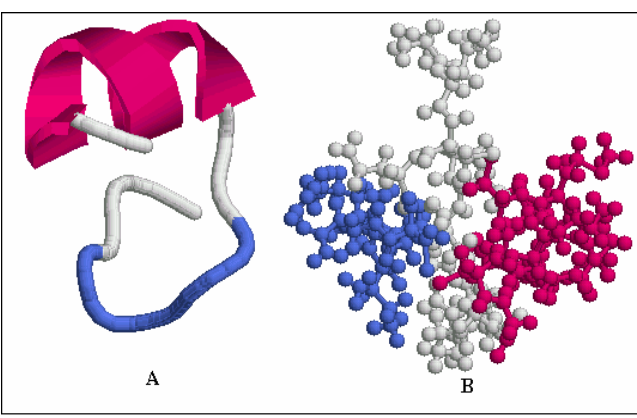

Figure 4-Structure of tertiapin of Apis mellifera [13]. Structure A showing the helical region in red (12-19 $A A$ ) and turn region in blue color (59 AA). Structure B showing boll and stick model, which show helical region in red color and turn in blue.

\section{Discussion}

For the prediction of antigenic determinant site of Tertiapin protein of Apis mellifera, we got one (1) antigenic determinant site in the sequence. The highest pick is recorded between AA 4 to AA 16. The sequence of AA in this region is 'NCNRIIIPHMCWK'. The average propensity for the tertiapin protein is found to be above 1.0697 (figure -1). All residues having above 1.0 propensity are always potentially antigenic (Kolaskar and Tongaonkar 1990). A region (4-16) preferably select peptides lying in loops connecting Secondary Structure (SS) motifs, avoiding peptides located in helical regions. According to Kyte-Doolittle, HoppWoods plot we can predict that sequence'NCNRIIIPHMCWK' is hydrophobic in nature. Predicted antigenic epitope is choosing peptides that are in the $\mathrm{C}$-terminal region of the tertiapin protein. Because the $\mathrm{N}-$ and Cterminal regions of proteins are usually solvent accessible and unstructured, antibodies against those regions are also likely to recognize the native protein. These regions are antigenic in nature and form antibodies. To improve immunogenicity, the peptides could be chemically conjugated to a large carrier protein. However, the process of chemical conjugation is not very reproducible, and uniformity of the peptide density on the carrier protein cannot be ensured. Recent findings show that peptides presented in a particulate form result in enhanced immune responses. One of the major treatments is gene therapy or recombinant DNA vaccines involve targeting multiple antigenic components to direct and empower the immune system to protect the host from chemical reaction. Antigenic epitopes of Tertiapin protein of Apis mellifera are important determinants. The knowledge of the immune responses to a protein antigen progressed, it became clear that the whole protein is not necessary for raising the immune response, but small segments of protein called the antigenic determinants or the epitopes are sufficient for eliciting the desired immune response. The predicted segment in tertiapin protein is 'NCNRIIIPHMCWK' and it should be 
tried on gel separation to get a pure form of it for primer prediction in human.

\section{Abbreviation}

RA- Rheumatoid Arthritis

$A A$, aa -Amino acid

\section{References}

[1] Miroshnikov A.I.; Boikov V.A.; Snezhkova L.G.; Severin S.E.; Shvets V.I. (1983) Bioorg Khim., 9(1), 26-32.

[2] Dudkin S.M.; Mikchaylova L.I.; Severin E.S. Jr. (1983) Adv Enzyme Regul., 21, 333-52.

[3] Jin W.; Klem A.M.; Lewis J.H.; Lu Z. (1999) Biochemistry, 38(43),14294301.

[4] Kitamura H.; Yokoyama M.; Akita H.; Matsushita K.; Kurachi Y.; Yamada M. (2000) J Pharmacol Exp Ther., 293(1), 196-205.

[5] Jin W. and Lu Z. (1998) Biochemistry, 37(38), 13291-9.

[6] Ovchinnikov Y.A.; Miroshnikov A.I.; Kudelin A.B.; Kostina M.B.; Boikov V.A.; Magazanik L.G. and Gotgilf I.M. (1980) Bioorg. Khim., 6, 359-365.

[7] Benson D.A.; Karsch-Mizrachi I.; Lipman D.J.; Ostell J.; Wheeler D.L. (2003) Nucleic acids research, 31, 23.

[8] Bairoch A.; Apweiler R.; Wu C.H.; Barker W.C.; Boeckmann B.; Ferro S.; Gasteiger E.; Huang H.; Lopez R.; Magrane M.; Martin M.J.; Natale D.A.; O'Donovan C.; Redaschi N.; Yeh L.S. (2005) Nucleic Acids Res., 33(Database issue), D154-9.

[9] Pearson W.R. (1988) PNAS, 85, 2444.

[10] Kolaskar A. and Tongaonkar A (1990) FEBS Lett., 276(1-2), 172-4.

[11] Kyte J. and Doolittle R.F. (1982) J. Mol. Biol., 157, 105.

[12] Hoop T.P. and Woods K.R. (1978) Proc Natl Acad Sci USA., 78, 3824.

[13] Xu X.; Nelson J. W. (1993) Proteins 1993, $17,124$. 